\title{
Reusable Surface Plasmon Resonance Immunosensor for Temporal Information of Protein Biomarkers
}

\author{
Koji Toma, ${ }^{1}$ Chisato Kishikawa, ${ }^{2}$ Takahiro Arakawa, ${ }^{1}$ and Kohji Mitsubayashi ${ }^{1,2 *}$ \\ ${ }^{1}$ Department of Biomedical Devices and Instrumentation, Institute of Biomaterials and Bioengineering, \\ Tokyo Medical and Dental University, 2-3-10 Kanda-Surugadai, Chiyoda-ku, Tokyo 101-0062, Japan \\ ${ }^{2}$ Graduate School of Medical and Dental Sciences, Tokyo Medical and Dental University, \\ 1-5-45 Yushima, Bunkyo-ku, Tokyo 113-8510, Japan
}

(Received September 7, 2020; accepted November 26, 2020)

Keywords: surface plasmon resonance, immunosensor, repeated measurement

Monitoring temporal changes in antigen levels, such as disease-related markers in medical care or airborne allergens in environmental medicine, has been increasing in importance because it allows one to notice the level exceeding a threshold in real time, which helps the early detection or prevention of diseases. In this study, we have developed a reusable immunosensor that exploits surface plasmon resonance (SPR) and a $\mathrm{pH}$-resistant protein, ORLA85, for rapid and repeated (semicontinuous) immunoassays. After the sensor development, a model experiment using mouse ( $\mathrm{mIgG}$ ) and anti-mouse (a-mIgG) antibodies was performed. In the experiment, the sensitivity of the developed SPR immunosensor was initially evaluated using various concentrations of a-mIgG. The limit of detection of the sensor for a-mIgG was $14.2 \mathrm{ng} / \mathrm{mL}(0.09 \mathrm{nM})$ and the dynamic range was $138-30000 \mathrm{ng} / \mathrm{mL}(0.86-187.5 \mathrm{nM})$. In 10 repeated measurements of a-mIgG, the sensor signals returned to the baseline after removing a-mIgG using an acidic solution with $\mathrm{pH} 1$. The measurement time was $15 \mathrm{~min}$ and the coefficient of variation of the sensor outputs was $7.5 \%$. These results indicated that the developed SPR immunosensor had potential for semicontinuous measurement, suggesting its application to monitoring systems for the early detection and prevention of diseases.

\section{Introduction}

Immunoassay is a method to detect the presence or measure the concentration of an antigen molecule by an antibody. Unlike enzymes, antibodies can bind to relatively large molecules, such as proteins, and this useful character makes immunoassay a leading method for disease diagnosis and the detection of pathogens. ${ }^{(1,2)}$

Two major immunoassays are lateral flow immunochromatographic assay (LFIA) and enzyme-linked immunosorbent assay (ELISA). ${ }^{(3-6)}$ In LFIA, antigens added dropwise to a cellulose membrane first react with Au colloid-labeled antibodies, then they are transported through the cellulose membrane and captured by other antibodies, which are immobilized in advance in test and control lines, to exhibit colored marks in the lines, where the color indicates "Corresponding author: e-mail: m.bdi@tmd.ac.jp https://doi.org/10.18494/SAM.2021.3070 
a positive or negative result. In ELISA, antigens are added dropwise to wells of a microtiter plate, where primary antibodies are immobilized in advance. Afterwards, enzyme-labeled secondary antibodies are added to the wells to bind to the antigens. Finally, a substrate for the enzyme is added to produce chromogenic molecules, and the antigen concentrations can be measured by the absorbance of the products. Both methods have many attractive advantages for practical use. LFIA gives a result within about 10 min by simply adding a sample solution from a patient dropwise onto a test strip. Since it does not require large or complicated equipment, LFIA is widely used for point-of-care testing. ${ }^{(7)}$ ELISA, on the other hand, requires more complex procedures with special facilities than LFIA. However, it allows the quantitative evaluation of antigen concentrations with high sensitivity and throughput.

A number of cutting-edge studies have been devoted to the development of immunosensors using a variety of detection methods, such as electrochemistry, ${ }^{(8,9)}$ acoustic waves, ${ }^{(10-12)}$ and optical waveguides. ${ }^{(13,14)}$ Despite the dramatic improvements in sensitivity that have been achieved by these approaches, little attention has been paid to the repeated use of sensors.

We focused on the importance of temporal information of the antigen concentration. For example, the monitoring of airborne house dust mite allergens in a residential or working environment and detecting high levels of these allergens in real time could alert people, preventing them from exposure to the allergens and allergic diseases. ${ }^{(15-18)}$ To achieve semicontinuous immunoassay, the sensor surface must be able to be regenerated repeatedly without losing its ability to capture antigens.

Previously, we developed a surface-acoustic-wave-based reusable immunosensor for the semicontinuous measurement of mite allergens. ${ }^{(19-21)}$ The sensor utilized a highly stable protein to anchor capture antibodies. This surface modification allowed repeated measurement of mite allergens with high reproducibility. In this study, we investigated the possibility of combining this surface modification method with surface plasmon resonance (SPR) for the development of a reusable SPR immunosensor. SPR is a collective oscillation of free electrons in a thin metal film, which is induced by incident light under a resonance condition, e.g., the incident angle of the light. By using the sensitivity of SPR to the refractive index in the vicinity of a metal surface, SPR spectroscopy is known to be a powerful technique for observing molecular binding to a metal surface. Herein, we developed an SPR immunosensor to provide temporal information of protein biomarkers and assessed its basic characteristics and the possibility of semicontinuous measurement using mouse and anti-mouse $\mathrm{IgG}$, which mimicked a sandwich assay.

\section{Materials and Methods}

\subsection{Materials}

Ethanolamine, $\beta$-mercaptoethanol (BME, M6250), 2,2'-azino-bis(3-ethylbenzothiazoline6-sulfonic acid) diammonium salt (ABTS, A1888), and bovine serum albumin (BSA, A2153) were purchased from Sigma-Aldrich (Japan). Sodium dodecyl sulfate (SDS, 199-07141), sodium acetate, and acetic acid were purchased from FUJIFILM Wako Pure Chemical (Japan). 
Mouse IgG (mIgG, 02-6502) and PEGylated bis(sulfosuccinimidyl)suberate (BS(PEG)5) were purchased from Themo Fisher Scientific (Japan). Polyclonal anti-mouse IgG (a-mIgG, anti-Fab of mIgG from goat, A90-100A) was obtained from Bethyl Laboratories (USA). Orla IgGBinder-G kit, including a mixed solution of protein G-fused Orla protein (ORLA85 protein) and 11-mercaptoundec-11-ylhexaethylene glycol (HS-C11-EG6, PEG-thiol), and tris(2carboxyethyl)phosphine (TCEP) and Tris-HCl buffer solution (50 mM, pH 7.5), was from Orla Protein Technologies (UK). Phosphate buffered saline (PBS) with Tween 20 (PBS-T) was prepared by adding Tween $20[0.05 \%(\mathrm{v} / \mathrm{v})]$ to PBS solution (10 mM phosphate buffer, $140 \mathrm{mM}$ $\mathrm{NaCl}, 3 \mathrm{mM} \mathrm{KCl}, \mathrm{pH}$ 7.4). Acetate buffered saline (ABS) was prepared by adding acetic acid solution $(10 \mathrm{mM})$ to sodium acetate solution $(10 \mathrm{mM})$ until the $\mathrm{pH}$ reached a desired value. A mixture of ABS and Tween 20 [ABS-T, 0.05\% (v/v) Tween in ABS] was prepared in the same way as PBS-T. Carbonate-bicarbonate buffer $(\mathrm{CB}, 80 \mathrm{mM})$ solution was prepared by buffering sodium hydrogen carbonate $(80 \mathrm{mM}$ in ultrapure water, Wako) solution to $\mathrm{pH} 9.5$ with sodium carbonate solution ( $80 \mathrm{mM}$ in ultrapure water, Wako). Horseradish peroxidase (HRP)labeled a-mIgG (HRP-a-mIgG) for the ELISA experiment was prepared by following the manufacturer's protocol with a conjugation kit (Abcam, ab102890).

\subsection{Construction of apparatus for SPR immunosensor}

The apparatus of the SPR immunosensor is presented in Fig. 1(a). It uses the attenuated total reflection method with the Kretschmann configuration to excite surface plasmon polaritons (SPPs). The excitation light from a helium-neon laser (HeNe; $5.0 \mathrm{~mW}, \mathrm{R}-30991$, Newport) at a wavelength of $633 \mathrm{~nm}$ passes through a linear polarizer (LP, LPVISE050-A, Thorlabs) to make the light p-polarized; the laser light is split by a beam splitter (BS, BP250, split ratio of 50:50 at $\lambda=633 \mathrm{~nm}$, Thorlabs). One light is incident to a photodiode detector (PD1, PDA100A-EC, Thorlabs) and the other is incident to a glass prism (S-LAH71, refractive index $n_{\text {glass }}=1.845$, Natsume Optical, Japan) with a Au substrate at incident angle $\theta$. The reflected light from the prism is detected by another photodiode detector (PD2). PD1 was used for a reference signal to

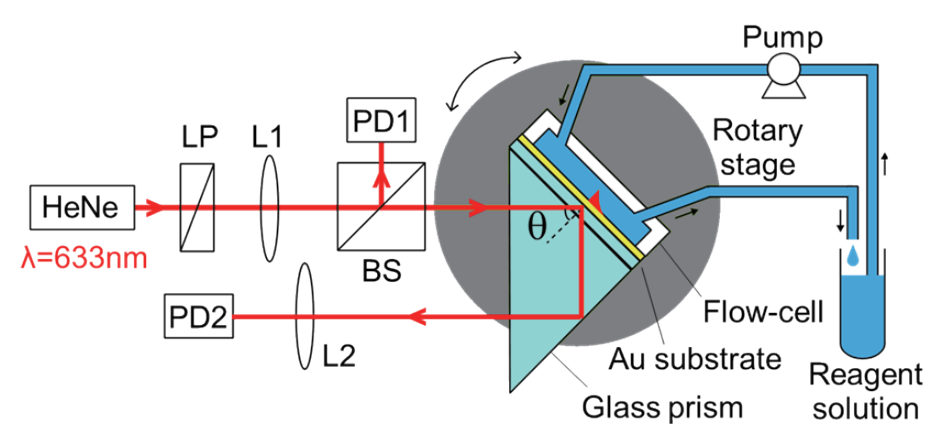

(a)

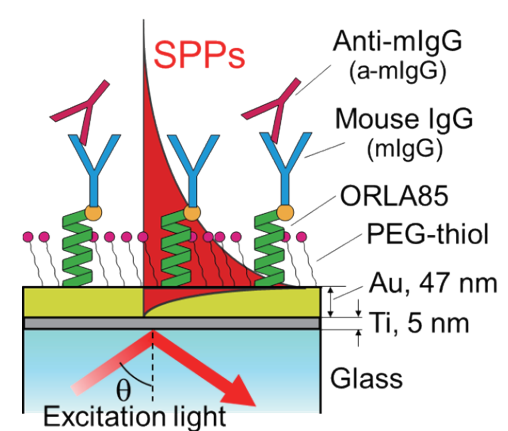

(b)

Fig. 1. (Color online) Schematic illustrations of (a) apparatus of SPR immunosensor and (b) Au substrate for immunosensor. HeNe, helium-neon laser; LP, linear polarizer; L, lens; PD, photodiode detector; BS, beam splitter. 
eliminate the environment-caused noises in the sample signal from PD2. The Au substrate was attached to the prism with a refractive-index-matching oil (1812X, Cargille Laboratories, USA) and covered with a flow cell that was connected to a peristaltic pump (ISM 596, ISMATEC, Switzerland) to run solutions.

The Au substrate used for SPP excitation was fabricated by successive sputtering of 4-nmthick Ti and 47-nm-thick Au on a glass substrate [see Fig. 1(b)]. The flow cell was made of poly(dimethylsiloxane) (PDMS; SILPOT 184, Dow Corning Toray). A mixture of curing agent and prepolymer with a volume ratio of 1:9 was cast onto a mold and cured for $2 \mathrm{~h}$ at $60{ }^{\circ} \mathrm{C}$. After that, the fabricated flow cell with a flow domain height of $200 \mu \mathrm{m}$ was bonded to a glass area on the Au substrate by plasma bonding using an atmospheric-pressure plasma (Aiplasma, Panasonic, Japan).

\subsection{Fabrication of SPR immunosensor chip}

The fabrication of the SPR immunosensor chip began with hydrophilic treatment by a selfassembled monolayer (SAM) of BME. BME assists the formation of an ORLA85 SAM and to maintain the $\beta$-sheet structure of the Orla protein scaffold by relaxing the highly energetic Au surface. ${ }^{(22)}$ Although BME was also adsorbed on the Au surface by $\mathrm{S}-\mathrm{Au}$ bonding, it was possible for ORLA85 to replace short hydrophilic thiols such as BME. ${ }^{(23)}$ The BEM solution $[1 \%$ $(\mathrm{v} / \mathrm{v})$ in ultrapure water] was cast onto the Au substrate and incubated for $5 \mathrm{~min}$. After rinsing with PBS, a mixture solution of ORLA85 and PEG-thiol, which had been mixed and reacted with TCEP solution ( $0.5 \mathrm{M}$ in Tris-HCl buffer solution) for $10 \mathrm{~min}$ just before use in order to reduce the disulfide bonds in ORLA85 to dithiols, was cast onto the Au substrate to form a SAM of ORLA85 and PEG-thiol. Here, PEG-thiol worked as a filler molecule to enhance the stability of ORLA85 and its anti-fouling property. After 30 min reaction, the solution on the Au substrate was exchanged with SDS solution $[1 \%(\mathrm{v} / \mathrm{v})$ in ultrapure water] and the substrate was left for 3 min. After rinsing the Au substrate with PBS, weakly bound ORLA85 and PEG-thiol were removed by $\mathrm{HCl}(100 \mathrm{mM}){ }^{(22,24,25)}$

Next, mIgG, imitating primary antibodies in the sandwich assay, was bound to the ORLA85modified Au substrate surface. To maximize the amount of $\mathrm{mIgG}$ on the surface, we performed two experiments to investigate the following: first, the influence of the buffer type and its $\mathrm{pH}$ on the binding efficiency of $\mathrm{mIgG}$ to protein $\mathrm{G}$; second, the influence of the concentration of mIgG solution. In the first experiment, a $100 \mu \mathrm{g} / \mathrm{mL} \mathrm{mIgG}$ solution was prepared with PBS-T or ABS-T with various $\mathrm{pH}$ values and left on the Au substrate for $15 \mathrm{~min}$ for $\mathrm{mIgG}$ to bind to protein G in ORLA85. In the second experiment, various concentrations $(3-300 \mu \mathrm{g} / \mathrm{mL})$ of $\mathrm{mIgG}$ solutions were prepared with an optimum buffer and its $\mathrm{pH}$ and left for $15 \mathrm{~min}$.

After the binding, mIgG and protein G of ORLA 85 were crosslinked by BS(PEG) 5 because this immobilization reduces the measurement time in repeated measurements by omitting the rebinding process of $\mathrm{mIgG}$. $\mathrm{BS}(\mathrm{PEG})_{5}$ solution $(0.5 \mathrm{mM}$ in PBS-T) was left for $10 \mathrm{~min}$, then $\mathrm{HCl}$ was added to remove uncrosslinked $\mathrm{mIgG}$ from the surface. Afterwards, an ethanolamine solution (1 M in ultrapure water, $\mathrm{pH} 8.4$ ) was applied and left for $15 \mathrm{~min}$ to passivate unreacted $N$-hydroxysuccinimide esters of $\mathrm{BS}(\mathrm{PEG})_{5}$. 


\subsection{Application to semicontinuous measurement of a-mIgG}

The sensitivity and the possibility of the semicontinuous measurement of a-mIgG were investigated for the developed SPR immunosensor. Figure 2 shows processes of repeated measurement: the a-mIgG solution prepared with PBS-T was loaded on the mIgG-immobilized sensor surface for $10 \mathrm{~min}$. After immune complex formation, a-mIgG was dissociated by $\mathrm{HCl}$ $(\mathrm{pH} 1)$ for surface regeneration, then the next measurement took place.

\subsection{Control experiment by ELISA}

As a reference experiment, ELISA was used to measure a-mIgG. First, $100 \mu \mathrm{L}$ of mIgG solution $(2 \mu \mathrm{g} / \mathrm{ml}$ in $\mathrm{CB})$ was added to each well of a microtiter plate, then the plate was incubated for $16 \mathrm{~h}$ at $4{ }^{\circ} \mathrm{C}$ for $\mathrm{mIgG}$ to adsorb on the well surface. Afterwards, BSA solution $(0.1 \mathrm{w} / \mathrm{w} \%$ in PBS-T) was added to the wells and left for $1 \mathrm{~h}$ to suppress the nonspecific binding of molecules. Next, various concentrations of HRP-a-mIgG $(0.5-250 \mathrm{ng} / \mathrm{mL}$ in PBS-T) were added in the wells and incubated for $1 \mathrm{~h}$ at room temperature. Finally, ABTS solution was added in the wells to start an HRP-catalyzed reaction. The reaction was terminated by SDS $(1 \mathrm{w} / \mathrm{v} \%$ in ultrapure water), and the absorbance at $405 \mathrm{~nm}$ wavelength was measured by a microplate reader (SH-1000Lab, Corona Electric, Japan). Note that the wells were rinsed with PBS-T every time before adding the reagents.

\section{Results and Discussion}

\subsection{Optimizations of SPR immunosensor}

A typical angular reflectivity spectrum of the Au substrate is shown in Fig. 3(a). The kink, found at the incident angle $\theta$ of $47.25 \mathrm{deg}$, corresponds to the total internal reflection angle $\theta_{\mathrm{c}}$ between the glass and buffer solution. The reflectivity dip, found at $\theta \sim 56.3 \mathrm{deg}$, represents the

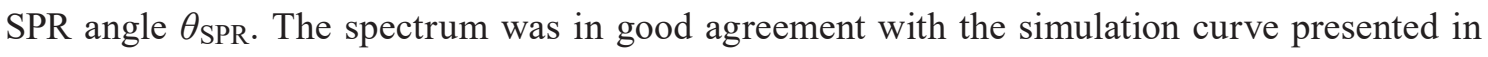
the same figure. The simulation was based on the Fresnel equations and conducted for a planar

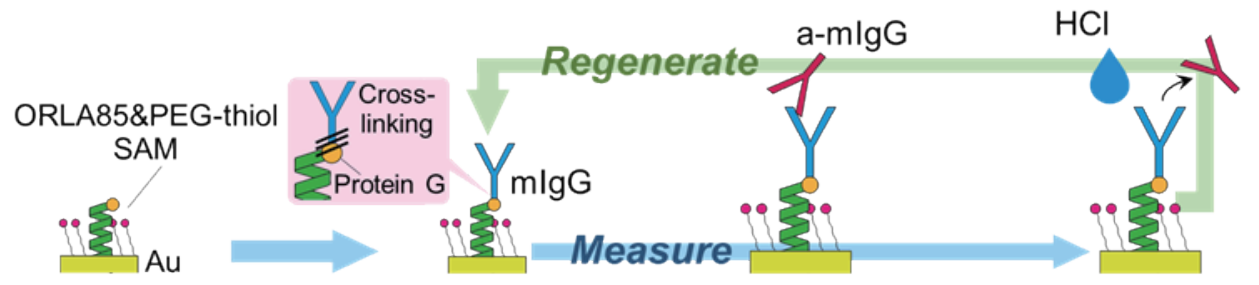
(i) Surface modification
of Au substrate
(ii) $\mathrm{mlgG}$
immobilization
(iii) Formation of
(iv) Removal of a-mlgG

Fig. 2. (Color online) (i, ii) Processes of fabrication of SPR immunosensor chip and (iii, iv) repeated measurement of a-mIgG. 


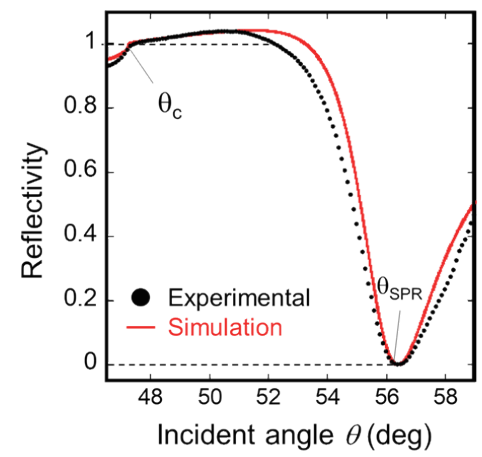

(a)

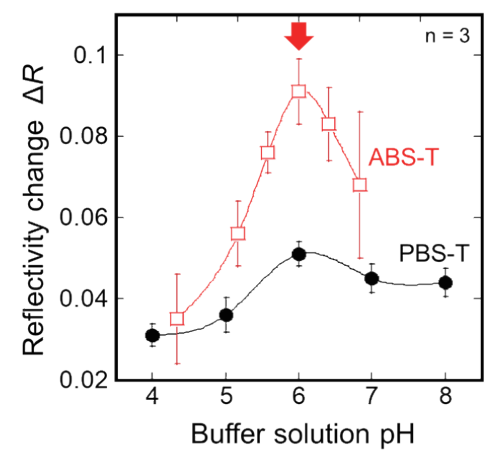

(b)

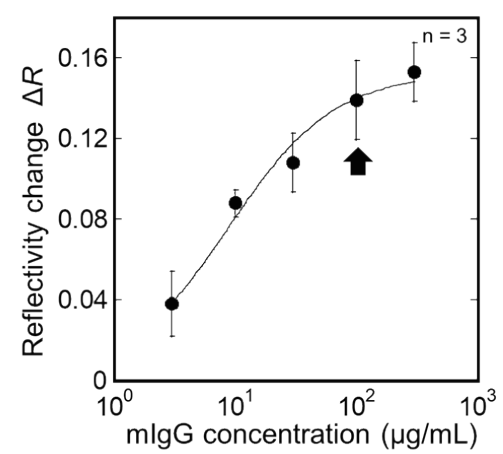

(c)

Fig. 3. (Color online) (a) Angular reflectivity spectrum of Au substrate. Effects of (b) buffer pH and (c) mIgG concentration on the $\mathrm{mIgG}$ binding to ORLA85.

multilayer structure model comprising glass $\left(n_{\text {glass }}=1.845\right)$, Ti (thickness $=5 \mathrm{~nm}, n_{\mathrm{Ti}}=2.053$ $+2.286 i),{ }^{(26)} \mathrm{Au}$ (thickness $\left.=47 \mathrm{~nm}, n_{\mathrm{Au}}=0.1431+3.610 i\right),{ }^{(27)}$ and water $\left(n_{\text {water }}=1.332\right)$. The binding of mIgG to ORLA85 was observed by a change in the reflectivity at the fixed angle where the initial relative reflectivity was $30 \%$.

The changes in the reflectivity $(\Delta R)$ upon the mIgG binding in ABS-T or PBS-T solution at various $\mathrm{pH}$ values are presented in Fig. 3(b). In both buffer solutions, $\Delta R$ increased with increasing $\mathrm{pH}$ and showed a peak at $\mathrm{pH}$ 6.0. This result, showing that the strongest binding between protein $\mathrm{G}$ and IgG occurred under a slightly acidic condition, agrees with previous studies. ${ }^{(19,20,28)}$

The dependence of the binding on the $\mathrm{mIgG}$ concentration was investigated, and it was found that more binding occurred with increasing concentration [see Fig. 3(c)]. Considering the reagent consumption and its effect, we decided to use $100 \mu \mathrm{g} / \mathrm{mL} \mathrm{mIgG}$ in subsequent experiments.

After mIgG binding to ORLA85, immobilization of $\mathrm{mIgG}$ was conducted. The change in the reflectivity during the binding and immobilization is presented in Fig. 4. The initial baseline was taken in PBS-T, and the reflectivity dropped when the solution was exchanged with ABS-T, a solvent for $\mathrm{mIgG}$, because of the refractive index difference between PBS-T and ABS-T. Afterwards, the binding of $\mathrm{mIgG}$ induced the increase in the reflectivity. When $\mathrm{BS}(\mathrm{PEG})_{5}$ was applied, the reflectivity dropped due to the refractive index difference between the solutions and the dissociation of $\mathrm{mIgG}$ in PBS-T, as expected from Fig. 3(a). After rinsing $\mathrm{BS}(\mathrm{PEG})_{5}$ with PBS-T, weakly immobilized $\mathrm{mIgG}$ was removed by $\mathrm{HCl}$ and further rinsing with PBS-T. We defined two changes in reflectivity, $\Delta R_{\text {before }}$ and $\Delta R_{\text {after, }}$, to evaluate bound and immobilized $\mathrm{mIgG}$, respectively. Calculating their ratio, $\Delta R_{\mathrm{after}} / \Delta R_{\text {before, }}$ we obtained the immobilization rate, which indicates the amount of immobilized $\mathrm{mIgG}$ relative to the already bound $\mathrm{mIgG}$. The immobilization rate was $84.3 \%$ in the first immobilization and increased to $96.3 \%$ upon repeating this process three times. Therefore, we immobilized $\mathrm{mIgG}$ by repeating the immobilization process three times. 


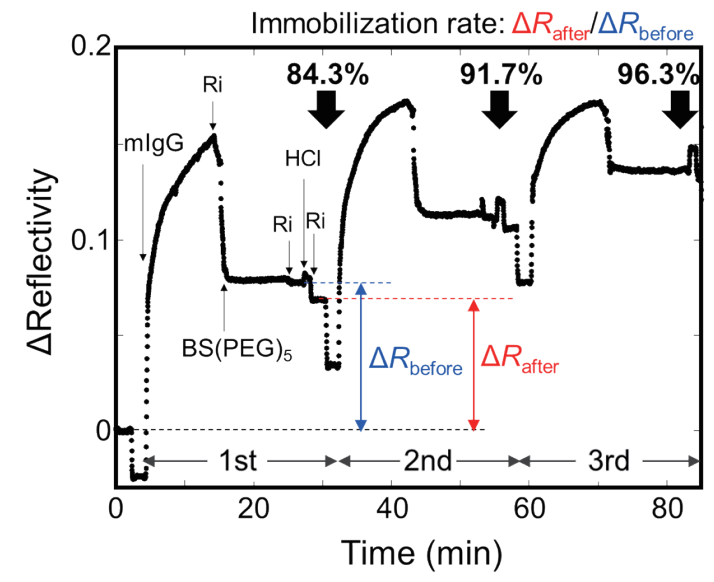

Fig. 4. (Color online) Change in reflectivity during $\mathrm{mIgG}$ immobilization.

\subsection{Sensitivity of SPR immunosensor and application to semicontinuous measurement of a-mIgG}

The sensitivity of the SPR immunosensor was investigated with various concentrations of a-mIgG. The sensor responses to a blank sample and 3 and $30 \mu \mathrm{g} / \mathrm{mL}$ of a-mIgG are presented in Fig. 5(a), which shows the change in the reflectivity at a fixed incident angle where the initial reflectivity was about $30 \%$. The reflectivity increased as a-mIgG was captured by $\mathrm{mIgG}$ on the $\mathrm{Au}$ surface because the presence of a-mIgG increases the local refractive index, which causes the shift of the SPR angle toward a higher angle. The change in reflectivity became larger with increasing concentration of a-mIgG, showing a clear correlation with the molecular concentration. Here, the sensor output, $\Delta R_{\mathrm{a}-\mathrm{mIgG}}$, was defined as the difference between the reflectivity after rinsing and the baseline. In addition, surface regeneration by $\mathrm{HCl}$ at the end of each measurement recovered the reflectivity to the baseline. This implies the possibility of repeated measurements of a-mIgG.

The relation between the sensor output of the SPR immunosensor and the a-mIgG concentration is plotted in Fig. 5(b). Here, the relation for ELISA is also plotted for comparison, and these plots were fitted by the following equations:

$$
\begin{gathered}
\text { SPR: } \Delta R_{\mathrm{a}-\mathrm{mIgG}}=A_{1}+\left(B_{1}-A_{1}\right) /\left\{1+\left(C_{1} /[\mathrm{a}-\mathrm{mIgG}(\mu \mathrm{g} / \mathrm{mL})]\right)^{D_{1}}\right\}, \\
\text { ELISA: Absorbance }=A_{2}+\left(B_{2}-A_{2}\right) /\left\{1+\left(C_{2} /[\mathrm{a}-\mathrm{mIgG}(\mu \mathrm{g} / \mathrm{mL})]\right)^{D_{2}}\right\},
\end{gathered}
$$

where $A_{1}=0.00051, B_{1}=0.054, C_{1}=4.9$, and $D_{1}=0.91$ are the coefficients for the SPR immunosensor with a coefficient of correlation $(R)$ of $0.998 ; A_{2}=0.11, B_{2}=5.9, C_{2}=0.56$, and $D_{2}=1.04$ are the coefficients for ELISA with $R$ of 0.999 ; and [a-mIgG] is the concentration of a-mIgG in $\mu \mathrm{g} / \mathrm{mL}$. From the calibration curves, the dynamic ranges and the limits of detection (LODs) are determined to be $138-30000 \mathrm{ng} / \mathrm{mL}(0.86-187.5 \mathrm{nM})$ and $14.2 \mathrm{ng} / \mathrm{mL}(0.09 \mathrm{nM})$ for the SPR immunosensor and 3.3-300 $\mathrm{ng} / \mathrm{mL}(0.021-1.88 \mathrm{nM})$ and $1.3 \mathrm{ng} / \mathrm{mL}(0.008 \mathrm{nM})$ 


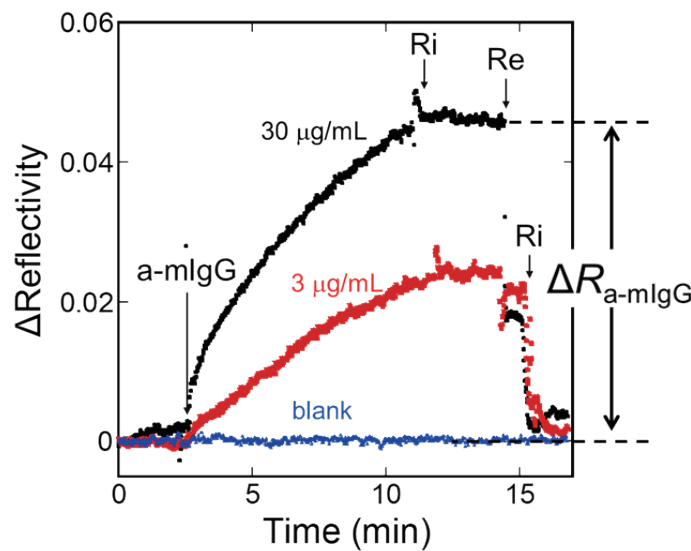

(a)

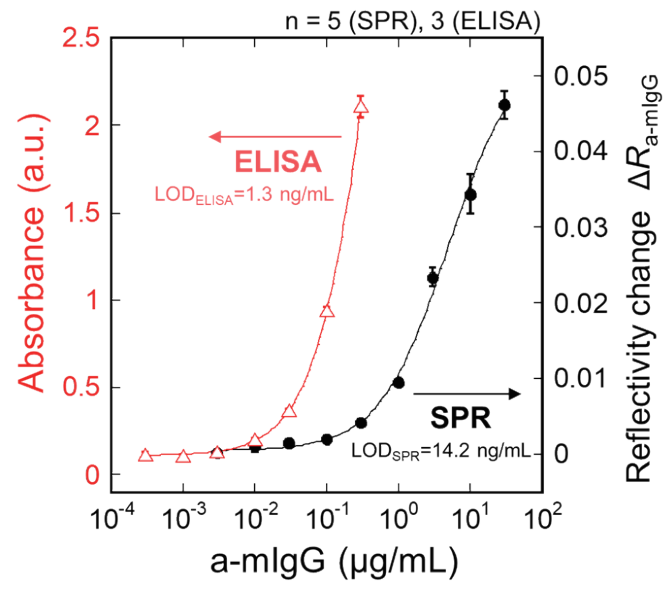

(b)

Fig. 5. (Color online) (a) Sensor responses to various concentrations of a-mIgG. (b) Calibration curves of SPR immunosensor and ELISA.

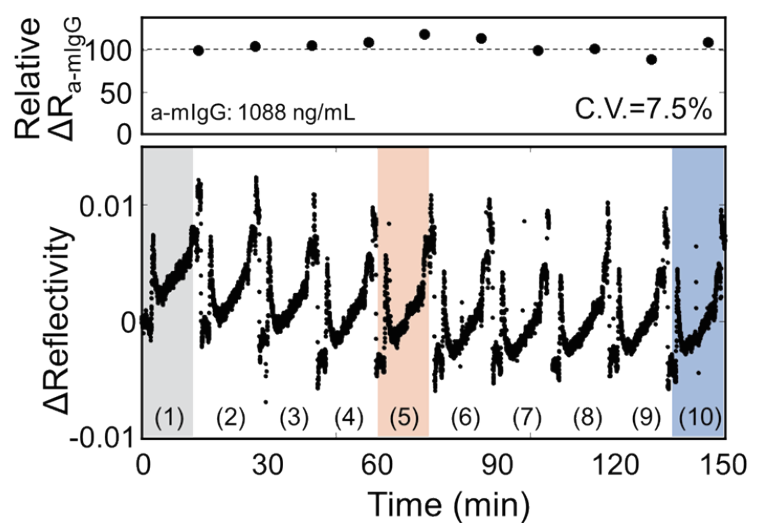

(a)

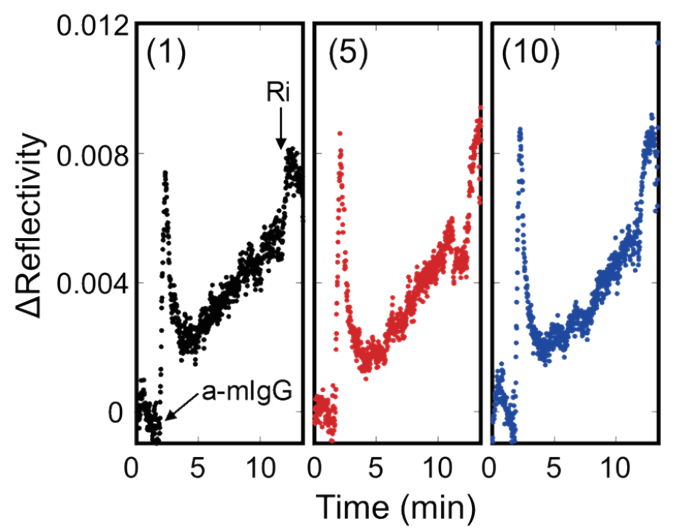

(b)

Fig. 6. (Color online) (a) Sensor responses to 10 repeated measurements of a-mIgG. (b) the first, fifth, and tenth measurements with aligned baselines.

for ELISA, respectively. Here, the limit of quantification and LOD were determined as the concentration where ten or three times the standard deviation of the reflectivity for the blank sample was equal to the sensor output. Although the sensitivity of the SPR immunosensor was not superior to that of ELISA, the measurement time was $15 \mathrm{~min}$, which was shortened by a factor of ten.

Finally, we investigated the possibility of semicontinuous measurement of a-mIgG. Figure 6(a) shows sensor responses to 10 repeated measurements of $1088 \mathrm{ng} / \mathrm{mL}(6.8 \mathrm{nM})$ a-mIgG. Throughout the measurements, we observed a negative baseline drift. However, as shown in Fig. 6(b), the sensor responses of the first, fifth, and tenth measurements were in good agreement when the baselines were aligned. The reproducibility of the sensor output was also high, as 
indicated by the coefficient of variation (C.V.) of the output of 7.5\%. The observed baseline drift may have been due to the dissociation of $\mathrm{mIgG}$ through the surface regeneration by $\mathrm{HCl}$. Despite this, the amount of residual $\mathrm{mIgG}$ on the sensor surface was sufficient relative to the applied amount of a-mIgG, suggesting that the measurements could be repeated with good reproducibility.

\section{Conclusions}

We have developed an SPR immunosensor for measuring temporal information of protein biomarkers. The sensor utilized a highly sensitive SPR and ORLA85, which has excellent $\mathrm{pH}$ resistance, to achieve stable primary antibody immobilization in repeated measurements and surface regeneration. Although the LOD of the SPR immunosensor of $14.2 \mathrm{ng} / \mathrm{mL}(0.09 \mathrm{nM})$ was inferior to that of ELISA of $1.3 \mathrm{ng} / \mathrm{mL}(0.008 \mathrm{nM})$, the measurement time of $15 \mathrm{~min}$ was shortened by a factor of ten from that of ELISA. In 10 repeated measurements of a-mIgG, the sensor outputs showed high reproducibility, as indicated by the C.V. of $7.5 \%$. These results suggest that the SPR immunosensor, which is capable of semicontinuous measurement, will be useful for monitoring the temporal information of biomarker concentrations.

\section{Acknowledgments}

This work was partly supported by the Japan Society for the Promotion of Science (JSPS, Japan) KAKENHI Grant Numbers JP 16K18112 and 18K13763; the Precise Measurement Technology Promotion Foundation (PMTP-F); Azuma Medical \& Dental Research Grant; and Ministry of Education, Culture, Sports, Science and Technology (MEXT, Japan) Special Funds for "Cooperative Research Project of Research Center for Biomedical Engineering".

\section{References}

1 P. E. Andreotti, G. V. Ludwig, A. H. Peruski, J. J. Tuite, S. S. Morse, and L. F. Peruski: Biotechniques 35 (2003) 850. https://doi.org/10.2144/03354ss02

2 T. R. J. Holford, F. Davis, and S. P. J. Higson: Biosens. Bioelectron. 34 (2011) 12. https://doi.org/10.1016/ j.bios.2011.10.023

3 K. A. Poehling, M. R. Griffin, R. S. Dittus, Y.-W. W. Tang, K. Holland, H. Li, and K. M. Edwards: Pediatrics 110 (2002) 83. https://doi.org/10.1542/peds.110.1.83

4 A. Tsay, L. Williams, E. B. Mitchell, and M. D. Chapman: Clin. Exp. Allergy 32 (2002) 1596. https://doi. org/10.1046/j.1365-2222.2002.01533.x

5 B. K. Van Weemen and A. H. W. M. Schuurs: FEBS Lett. 15 (1971) 232. https://doi.org/10.1016/00145793(71)80319-8

6 E. Engvall and P. Perlmann: Immunochemistry 8 (1971) 871. https://doi.org/10.1016/0019-2791(71)90454-X

7 P. B. Luppa, C. Müller, A. Schlichtiger, and H. Schlebusch: Trends Anal. Chem. 30 (2011) 887. https://doi. org/10.1016/j.trac.2011.01.019

8 Z. Wang, K. Shang, J. Dong, Z. Cheng, and S. Ai: Microchim. Acta 179 (2012) 227. https://doi.org/10.1007/ s00604-012-0874-6

9 M. Moreno-Guzmán, I. Ojeda, R. Villalonga, A. González-Cortés, P. Yáñez-Sedeño, and J. M. Pingarrón: Biosens. Bioelectron. 35 (2012) 82. https://doi.org/10.1016/j.bios.2012.02.015

10 M. Michalzik, J. Wendler, J. Rabe, S. Büttgenbach, and U. Bilitewski: Sens. Actuators, B 105 (2005) 508. https://doi.org/10.1016/j.snb.2004.07.012 
11 D. R. P. Morris, J. Fatisson, A. L. J. Olsson, N. Tufenkji, and A. R. Ferro: Sens. Actuators, B 190 (2014) 851. https://doi.org/10.1016/j.snb.2013.09.061

12 J. Lee, Y.-S. Choi, Y. Lee, H. J. Lee, J. N. Lee, S. K. Kim, K. Y. Han, E. C. Cho, J. C. Park, and S. S. Lee: Anal. Chem. 83 (2011) 8629. https://doi.org/10.1021/ac2020849

13 P. B. Luppa, L. J. Sokoll, and D. W. Chan: Clin. Chim. Acta. 314 (2001) 1. https://doi.org/10.1016/s0009$\underline{8981(01) 00629-5}$

14 J. Maldonado, A. B. González-Guerrero, C. Domínguez, and L. M. Lechuga: Biosens. Bioelectron. 85 (2016) 310. https://doi.org/10.1016/j.bios.2016.04.095

15 S. Lau, G. Falkenhorst, A. Weber, I. Werthmann, P. Lind, P. Buettner-Goetz, and U. Wahn: J. Allergy Clin. Immunol. 84 (1989) 718. https://doi.org/10.1016/0140-6736(92)91260-F

16 D. W. Hide, S. Matthews, L. Matthews, M. Stevens, S. Ridout, R. Twiselton, C. Gant, and S. H. Arshad: J. Allergy Clin. Immunol. 93 (1994) 842. https://doi.org/10.1016/0091-6749(94)90375-1

17 A. M. Warner, B. Bjbrksten, A. K. M. Munir, C. Möller, C. Schou, and N.-L. M. Kjellman: Pediatr. Allergy Immunol. 7 (1996) 61. https://doi.org/10.1111/j.1399-3038.1996.tb00108.x

18 J. Kuehr, T. Frischer, R. Meinert, R. Barth, J. Forster, S. Schraub, R. Urbanek, and W. Karmaus: J. Allergy Clin. Immunol. 94 (1994) 44. https://doi.org/10.1016/0091-6749(94)90070-1

19 K. Toma, D. Miki, C. Kishikawa, N. Yoshimura, K. Miyajima, T. Arakawa, H. Yatsuda, and K. Mitsubayashi: Anal. Chem. 87 (2015) 10470. https://doi.org/10.1021/acs.analchem.5b02594

20 K. Toma, Y. Harashima, N. Yoshimura, T. Arakawa, H. Yatsuda, K. Kanamori, and K. Mitsubayashi: Sens. Mater. 29 (2017) 1679. https://doi.org/10.18494/SAM.2017.1634

21 K. Toma, M. Horibe, C. Kishikawa, N. Yoshimura, T. Arakawa, H. Yatsuda, H. Shimomura, and K. Mitsubayashi: Sens. Actuators, B 248 (2017) 924. https://doi.org/10.1016/j.snb.2017.01.183

22 A. P. Le Brun, A. Soliakov, D. S. H. Shah, S. A. Holt, A. McGill, and J. H. Lakey: Biomed. Microdevices 17 (2015) 1. https://doi.org/10.1007/s10544-015-9951-Z

23 S. Terrettaz, W.-P. Ulrich, H. Vogel, Q. Hong, L. G. Dover, and J. H. Lakey: Protein Sci. 11 (2002) 1917. https:// doi.org/10.1110/ps.0206102

24 A. P. Le Brun, D. S. H. Shah, D. Athey, S. A. Holt, and J. H. Lakey: Int. J. Mol. Sci. 12 (2011) 5157. https://doi. org/10.3390/ijms12085157

25 A. P. Le Brun, S. A. Holt, D. S. H. Shah, C. F. Majkrzak, and J. H. Lakey: Biomaterials 32 (2011) 3303. https:// doi.org/10.1016/j.biomaterials.2011.01.026

26 K. J. Palm, J. B. Murray, T. C. Narayan, and J. N. Munday: ACS Photonics 5 (2018) 4677. https://doi. org/10.1021/acsphotonics.8b01243

27 K. M. McPeak, S. V. Jayanti, S. J. P. Kress, S. Meyer, S. Iotti, A. Rossinelli, and D. J. Norris: ACS Photonics 2 (2015) 326. https://doi.org/10.1021/ph5004237

28 B. Akerström and L. Björck: J. Biol. Chem. 261 (1986) 10240. 